\title{
Serological Estimation, Molecular Detection and Serotyping of Dengue Virus in Dengue Patients of Lahore Region
}

\author{
Nusrat Bano", Muhammad Tayyab ${ }^{1 *}$, Muhammad Pervaiz ${ }^{2}$, Nosheen Yousaf ${ }^{3}$, \\ Ali Raza Awan', Abu Saeed Hashmi', Shehla Bhatti ${ }^{4}$, Maryam Hameed ${ }^{4}$, \\ Muhammad Wasim ${ }^{1}$, Sehrish Firyal ${ }^{1}$, Shagufta Saeed ${ }^{1}$, \\ Zaheer Hussain ${ }^{5}$, Munawar Iqbal ${ }^{6}$, Umer Younas ${ }^{6 * *}$ \\ ${ }^{1}$ Institute of Biochemistry and Biotechnology, University of Veterinary and Animal Sciences, Lahore, Pakistan \\ ${ }^{2}$ Department of Chemistry, Khwaja Fareed University of Engineering \& Information Technology, \\ Rahim Yar Khan, Pakistan \\ ${ }^{3}$ Pathology Department, Allama Iqbal Medical College, Lahore, Pakistan \\ ${ }^{4}$ Adults Emergency, Mayo Hospital, Lahore, Pakistan \\ ${ }^{5}$ Institute of Agricultural Sciences, University of the Punjab, Quaid-e-Azam Campus, Lahore, Pakistan \\ ${ }^{6}$ Department of Chemistry, The University of Lahore, Lahore, Pakistan
}

Received: 15 October 2020

Accepted: 24 March 2021

\begin{abstract}
Dengue has become endemic in Pakistan, and increasing cases of dengue have been reported every year since 2006. The objective of the study was to serologically and molecularly diagnose dengue disease and to identify the circulating serotypes in patients of the Lahore region. Total 345 blood samples were collected from Jinnah Hospitals Lahore (JHL) and Mayo Hospital Lahore (MHL). Dengue specific antigen and antibodies were detected in samples by two serological methods namely, immunochromatographic test device (ICT) and Enzyme-linked immunosorbent assay (ELISA). Acute phase samples were further subjected to molecular detection and serotyping of dengue virus by nested reverse transcriptase-polymerase chain reaction (RT-PCR). Similarity and divergence studies and the phylogenetic relationship was conducted on obtained sequences. Out of 345 samples subjected to serological analysis, 114 (33\%) were positive to dengue by ICT method while $110(31.8 \%)$ were detected positive by ELISA. Total $108(31.3 \%)$ samples were confirmed dengue positive by both serological methods, out of which 39 (36.1\%) samples were NS1 positive by both ICT and ELISA methods. By nested RT-PCR of 39 NS1 positive samples, the dengue virus was detected in $8(20.5 \%)$ patients. In this study DENV-1 was detected in 1(12.5\%) sample, DENV-2 in 3 (37.5\%) samples and DENV-3 in 4 (50\%) samples out of 8 PCR positive samples. The DENV-3 was the prevalent serotype. Similarity studies and phylogenetic analysis conducted on three serotypes (DENV-1, DENV-2 \& DENV-3) revealed their
\end{abstract}

\footnotetext{
*e-mail: muhammad.tayyab@uvas.edu.pk
}

**e-mail: umer.younas@chem.uol.edu.pk 
relatedness with dengue isolates from Sri Lanka, Singapore and India. This study demonstrated the prevalence of DENV-3 serotype in Lahore region in 2013 that is unique from the previously reported dengue serotypes in Pakistan. Moreover, this study concluded that both selected serological methods (ICT and ELISA) are equally good and comparable. The diagnostic frequency could efficiently be increased by detecting NS1 antigen along with $\operatorname{IgM} / \operatorname{IgG}$ antibodies.

Keywords: Dengue Virus, serotyping, NS1 antigen, RT-PCR, phylogenetic analysis

\section{Introduction}

Dengue fever caused by dengue virus (DENV) is one of the most important arthropods born arboviral diseases that poses threats to more than two-fifth of world population. It belongs to family Flaviviridae and genus Flavivirus and has four serotypes referred to as, DENV-1 to DENV-4 that are genetically similar but antigenically distinct. The major vectors responsible for dengue virus transmission in tropical and subtropical regions of the world are female mosquitoes of genus Aedes aegypti and Aedes albopictus [1,2]. Dengue is expanding in more than 125 countries located in tropical and subtropical regions of the world [3, 4]. Explosive population growth, unplanned urban inhabitants, with inadequate public health systems, increased international recreational, viral evolution and military travel to endemic areas are the major factor responsible for spread of disease $[5,6]$.

Dengue fever has been classified by World Health Organization (WHO) into dengue fever (DF), dengue hemorrhagic fever (DHF) and dengue shock syndrome (DSS) according to the severity of disease. Although dengue virus infection results in broad range of clinical symptoms, early and accurate laboratory diagnosis and serotyping of circulating dengue virus is essential for better and appropriate clinical management of patient and for proper control of epidemic by the concerned authorities in time [7].

Pakistan has been suffering from dengue outbreaks for decades. Its climatic condition and subtropical location are suitable for expansion of vectors as a result many vectors borne diseases may exist in this region [8]. The first dengue epidemic in Pakistan was reported in 1994 by which dengue serotype DENV-1 and DENV-2 were isolated [9]. The dengue remerged in Karachi in 2005, decade after the first epidemic and serotype DENV-3 was found to be involved. During 2006-2008 outbreaks of DENV-1,2 and 3 were responsible for 9900 cases with 79 deaths in multiple districts of Pakistan [9]. One of the worst out-break have been reported during the period of 2010 and 2011 as Pakistan faced adverse floods that increased breeding sites for dengue vector. Maximum dengue cases (17493) with 290 deaths were reported from Lahore, Punjab [10].

Due to repeated dengue outbreaks, current study was designed to diagnose disease by two serological methods and determine dengue serotypes by applying molecular techniques. The obtained sequences were aligned with global sequences retrieved from GenBank to determine phylogenetic relationship and geographic origin of circulating dengue virus isolates. Accurate and early diagnosis and molecular typing of circulating viruses can be helpful for patient treatment and effective dengue control.

\section{Materials and Methods}

\section{Sample Collection}

Total 345 suspected cases, visiting Jinnah hospital Lahore and Mayo hospital Lahore were enrolled for this study, during a period of one year from January 2013 to December 2013. Clinically suspected patients with more than two days of acute febrile illness along with two of the symptoms of headache, retro orbital pain, myalgia, arthralgia, rash and hemorrhagic manifestations were included in the study as per criteria [11]. Patients with identified bacterial infection, any other specified chronic infection were not included in the study.

\section{Data Collection}

Suspected dengue patients, both male and female and age ranged between 10-70 years, were thoroughly checked by the physician and their details regarding name, age, sex, days of fever, previous history of dengue, and location of the patients were entered in Performa. Twenty persons with other febrile (nondengue) illness (OFI) were included in study as control. Blood samples of patients and control were collected aseptically, allowed to clot and centrifuged. The serum was separated and stored in duplicate in $1.0 \mathrm{ml}$ storage vials. One set of serum was stored at $4-8^{\circ} \mathrm{C}$ for serological diagnosis and other set of serum was stored at $-80^{\circ} \mathrm{C}$ for dengue virus RNA extraction and serotyping of dengue.

\section{Serology}

All the 345 samples were screened for dengue specific IgG and IgM antibodies and non-structural protein-1(NS1) antigen with two standard laboratory procedures as ICT [12, 13] and ELISA [14, 15] and results were compared.

Commercially available dengue immunochromatographic diagnostic kits for NS1 antigen, $\operatorname{IgM} / \mathrm{IgG}$ antibodies of SD BIOLINE, Standard Diagnostic Inc; 
Seoul, Korea, and commercially available ELISA kits of NS1 antigen, IgM and IgG antibodies, manufactured by (Diagnostic Automation/Cortez Diagnostics, California, USA) were used for detection of dengue virus. All samples were processed according to manufacturer protocol.

\section{Molecular Analysis}

Blood samples of 39 patients those were NS1 positive with both ICT and ELISA methods were used for molecular analysis as dengue virus is present only during acute phase of infection. The blood samples were subjected to nested RT-PCR for molecular characterization of dengue virus, using primers reported in previous study [16].

\section{RNA Extraction}

To study molecular typing, viral RNA was extracted from all 39 NS1 positive samples by commercial viral RNA extraction kit, Nucleospin (Macherey-Nagel, Germany) and was stored for further use at $-80^{\circ} \mathrm{C}$ [16].

\section{Complementary DNA Synthesis (cDNA)}

Eluted RNA was reverse transcribed to complementary DNA (cDNA) using commercial kit, RevertAid ${ }^{\mathrm{TM}}$ M-M LV Reverse Transcriptase (Fermentas, MA, USA) prior to enzymatic DNA amplification. Reaction tube with final reaction volume $20 \mu \mathrm{L}$, containing $10 \mu \mathrm{L}$ of extracted RNA, reaction mixture of $10 \mu \mathrm{L}$ containing $4 \mu \mathrm{L}$ of $5 \times$ RT buffer, $0.5 \mu \mathrm{L} 0.1 \mathrm{M}$ Dithiothriotol, $2 \mu \mathrm{L}$ of $10 \mathrm{mM}$ dNTPs, $1 \mu \mathrm{L} 20 \mathrm{pM}$ antisense primer D2-D5/TTGCACCARC ARTCWATGTCTTCWGGYTC-3', PCR grade water $1.5 \mu \mathrm{L}$ and $1 \mu \mathrm{L}$ (200 units) of $\mathrm{M}-\mathrm{MLV}$ reverse transcriptase enzyme, was incubated at $37^{\circ} \mathrm{C}$ for $60 \mathrm{~min}$ followed by inactivation at $95^{\circ} \mathrm{C}$ for $3 \mathrm{~min}$. The prepared cDNA was immediately used for amplification of viral genes.

\section{Serotype Specific PCR Amplification and Sequencing}

Resulting cDNA was subsequently subjected to $T a q$ polymerase amplification with dengue virus consensus upstream primer D1-D 5'-TCAATATGCTGAAACGCGCGAGAAACCG-3 and downstream primer D2-D 5'-TTGCACCARCARTCWATGTCTTCWGGYTC-31 Target cDNA was amplified in $50 \mu \mathrm{L}$ volume containing $25 \mu \mathrm{L}$ of prepared PCR reaction mixture $2 \mathrm{x}$ of Red Taq (Merck), $5 \mu \mathrm{L}$ cDNA as template, $1 \mu \mathrm{L} 20 \mathrm{pM}$ primers (D1-D and D2-D) [16] each, I $\mu \mathrm{L}$ Taq DNA polymerase (Invitrogen Biotechnologies USA), and $17 \mu \mathrm{L}$ of PCR grade water. DNA segment comprises of $511 \mathrm{bp}$, specific to all four dengue serotypes was amplified. Thermal profile for reaction includes, initial denaturation at $95^{\circ} \mathrm{C}$ for 3 min followed by 35 cycles of denaturation at $94^{\circ} \mathrm{C}$ for $45 \mathrm{~s}$, primer annealing at $52^{\circ} \mathrm{C}$ for $45 \mathrm{~s}$ and extension at $72^{\circ} \mathrm{C}$ for 2 min along with final extension at $72^{\circ} \mathrm{C}$ for $10 \mathrm{~min}$.

DENV serotyping was conducted by second round of PCR amplification using $10 \mu \mathrm{L}$ of diluted DNA (1:100) of initial amplification as template and four serotype specific sense and antisense primers reported previously [16] to amplify different serotypes of dengue virus. Second round thermal profile was similar to first round thermal profile but annealing was carried out at $52^{\circ} \mathrm{C}$ for $45 \mathrm{~s}$. PCR products of second round were electrophoresed through $1 \%$ pre stained agarose gel and viewed under ultraviolet light using a gel documentation system (Wealtec, USA). The corresponding amplified bands were extracted from the agarose gel and purified by using Gel extraction kit (GeneAll, Korea) [17]. The purified DNA samples were sequenced. The obtained sequences were analyzed by using BioEdit and submitted to GenBank for accession number. The sequences obtained in present study and other sequences retrieved from GenBank were utilized for homology and phylogenetic analysis.

\section{Homology Studies and Phylogenetic Analysis}

The DENV sequences obtained in this study were aligned together with various global reference sequences from GenBank, to produce a consensus sequence of partial C-prM gene in CLUSTAL W program [18]. Similarity and divergence were deducted by using MegAlign software version 5 (DNA Star, Inc., Madison, WI, USA). Phylogenetic analysis was conducted by using Mega 5.2 software [19]. Dengue viruses with accession number KX098540.1, HM626123.1 and KT180266.1 for Dengue virus serotype 1, 2, and 3 respectively were used as reference sequence.

\section{Results and Discussion}

Dengue prevalence was observed during monsoon and cases started from the month of July and attained highest peck in September and decline in November. Dengue cases were not reported during months of January to June. Male population was more affected $(74 \%)$ than female population (26\%). Frequency of dengue infection was high in population of age 10-40 years as compared to age group 50-80 years.

\section{Serological Finding}

During the study period, out of 345 samples collected, 114 samples (33\%) were positive by ICT method and 110 samples (31.8\%) were positive by ELISA to dengue NS1 antigen and immunoglobulin (IgM, IgG). Both RIC and ELISA diagnostic results for dengue NS1 antigen were comparable and equally good. Sensitivity and specificity of NS1 antigen ICT kit was 
$96.87 \%$ and $99.28 \%$ respectively taking ELISA as gold standard.

Total six samples (3NS1, 1 NS1 \& IgM and 2 IgM \& IgG) positive by RIC method were confirmed negative for dengue infection by ELISA, while two samples confirmed NS1 positive by ELISA were not diagnosed by ICT method and these samples were not included in study (eight samples excluded from study). Total 108 samples $(31.3 \%)$ were confirmed positive by both methods. Out of 108 samples, 39 (36.1\%) were positive to NS1 antigen.

\section{Molecular Analysis of Dengue Virus}

Total 39 acute phase samples that were positive to dengue NS1 antigen were subjected to nested RT-PCR to detect dengue virus serotypes using serotype specific primers. Eight samples (20.5\%) labelled from N1 to N8 were positive to DENV infection in this study. Out of these 8 DENV positive cases one sample (12.5\%) labelled as N4 was diagnosed as DENV-1serotype, three samples (37.5\%) labelled as N5, N7 and N8 were diagnosed as DENV-2 serotypes while four samples $(50 \%)$ labelled as N1, N2, N3, N6 were of DENV-3 serotypes. The size of amplified product was 411, 403 and 453 bp for serotypes DENV-1, DENV-2 and DENV-3 respectively. The amplified products were eluted from gel, purified and then sequenced.

The sequences deposited in GenBank database as following accession numbers; GenBank: MF095886 (DENV-1strain) GenBank: MF071298-MF 071300 (DENV-2 strains) and GenBank: MF071294-MF071297 (DENV-3).

\section{Homology Studies}

Similarity and divergence studies reflected that serotype 1 strain was close to China and Singapore strains of 2012-2014 outbreaks with an average of 99\% of homology while most divergence was observed with Cambodian and Saudi strains with only 92.3\% similarity. Serotype DENV-2 isolated in this study was found close to Indian strains of 1996 outbreak with $98.2 \%$ homology. Comparison of DENV-3 strains isolated in this study with rest of the world strains showed highest similarity with Senegal and China dengue virus 3 strains of 2009 outbreak.

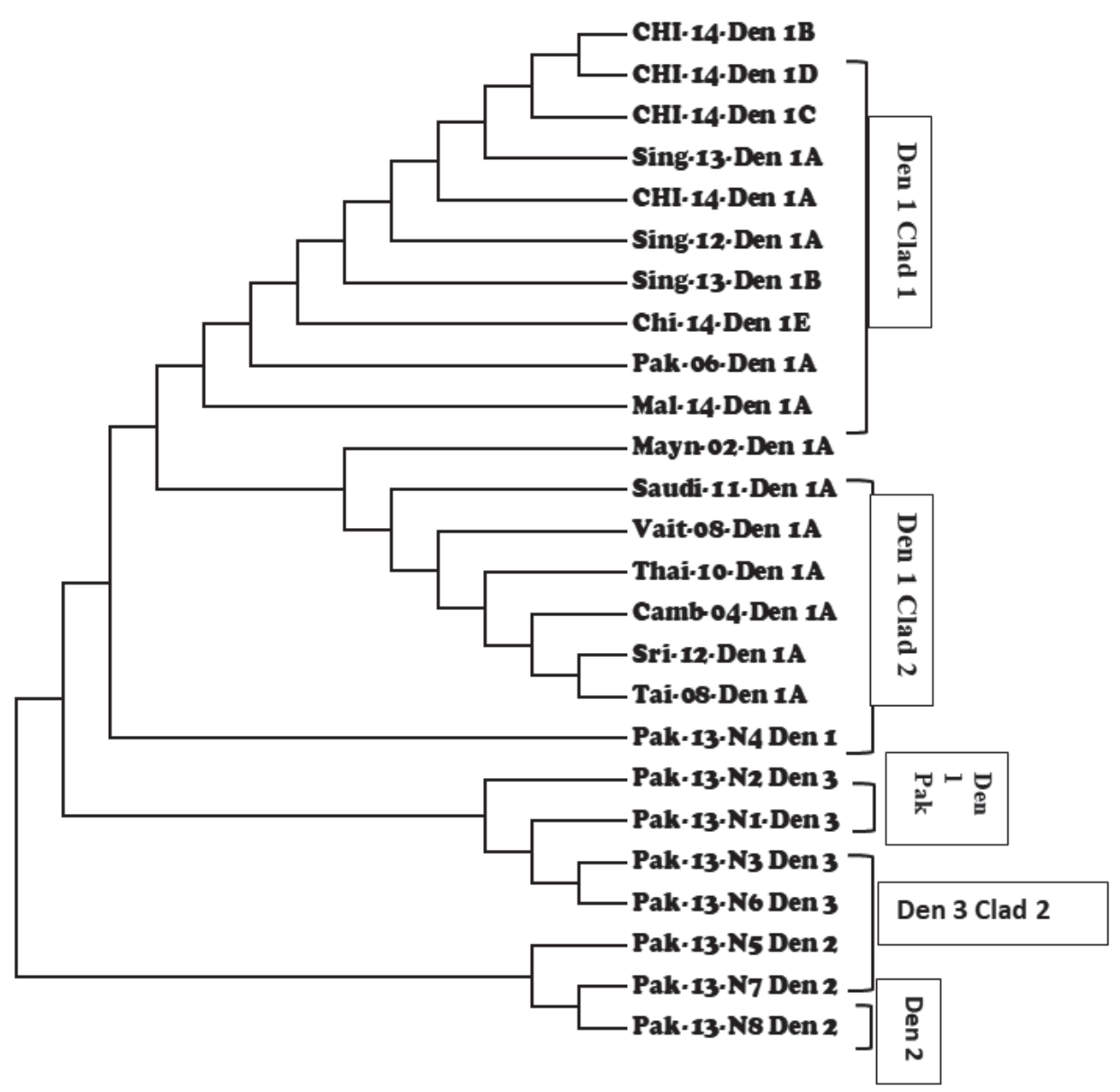

Fig. 1. Phylogenetic tree of dengue virus serotype 1 based on C-prM regions corresponding to nt125-529(404 bp) of the isolates in Lahore region in 2013. The one isolate DENV-1 was aligned with 24 reference sequences retrieved from GenBank. DENV-2 and DENV-3 sequences obtained in this study were used as out group. The tree was constructed by neighbor joining method using Mega 5.2 software. ${ }^{19}$ 


\section{Phylogenetic Analysis}

The C-prM genes sequences corresponding to nucleotide 25-529 (404 bp) of one DENV strain isolated in this study was aligned with twenty-five DENV-1 isolates retrieved from GenBank database. The isolated DEN-1 strain formed separate clad than other Pakistani DEN-1 strains that were clustered together with Chinese, Singaporean, Malaysian strains as shown in Fig. 1.

For DENV-2 strains, a sequence of 400 bp corresponding to C-prM region spanning from nucleotide 154-554 of 25 strains including three strains sequenced in this study were aligned for phylogenetic studies The Pakistani strain isolated in this study showed similarity with other dengue virus serotype 2 strains reported from 2008, 2009 and 2011 dengue outbreaks in Pakistan. The Pakistani dengue 2 isolate formed separate cluster (Den 2 clade 1) along with Indian strain as indicated in Fig. 2.

Phylogenetic analysis of 4 DENV-3 strains sequenced in this study was carried out by aligning C-prM region of other strains retrieved from GenBank database. The aligned region spanned from nucleotide 103-504 (401 bp) for DENV-3. Phylogenetic analysis indicated that Pakistani dengue 3 isolate formed separate clad near to Indian dengue virus that cause outbreak in 2012 as shown in Fig. 3.

Comparison of dengue NS1 antigen results by ICT with that of ELISA (taken as gold standard), showed that both methods were comparable and sensitivity and specificity of dengue NS1 (SD BIOLINE) kit calculated by Bayes theorem was $96.87 \%$ and $99.28 \%$ respectively. Total 39 samples of acute phase infection were positive to NS1 antigen by both methods. It is known that NS1 antigen appears early in dengue infection so diagnostic frequency could significantly be increased by using dengue NS1 Ag detection in combination with $\operatorname{IgM} / \operatorname{IgG}$ detection. The similar finding was observed in another study that dengue NS1 antigen detection in combination with anti-glycoprotein $\operatorname{IgM}$ and $\operatorname{IgG}$ serology could significantly increase the sensitivity of acute dengue diagnosis [20].

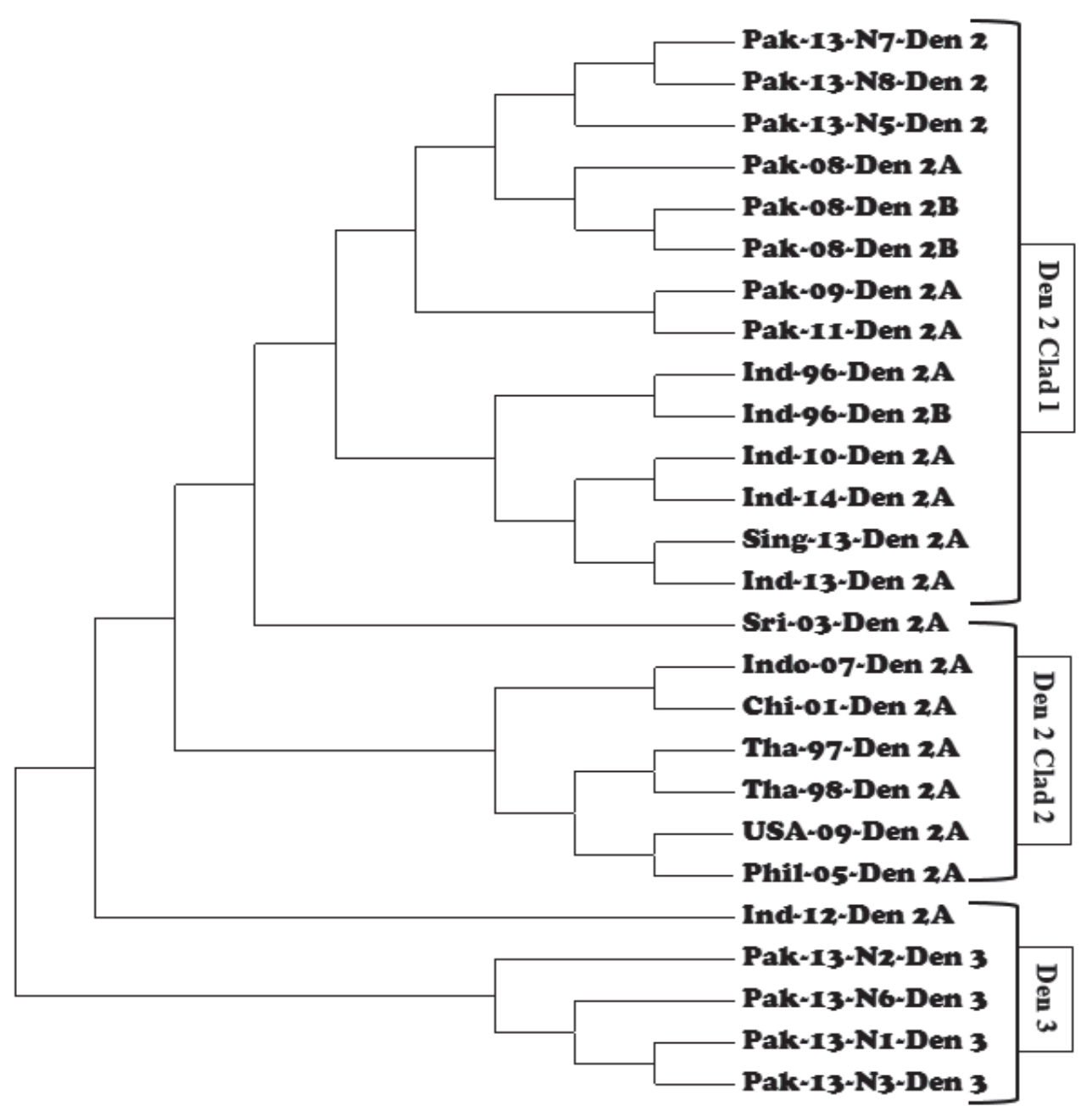

Fig. 2. Phylogenetic analysis of dengue virus serotype 2 based on C-prM regions corresponding to nt154-554(400 bp) of the isolates in Lahore region in 2013. The three isolates of DENV-2 were aligned with reference sequences retrieved from GenBank. The tree was constructed by neighbor joining method using Mega 5.2 software. ${ }^{19}$ 


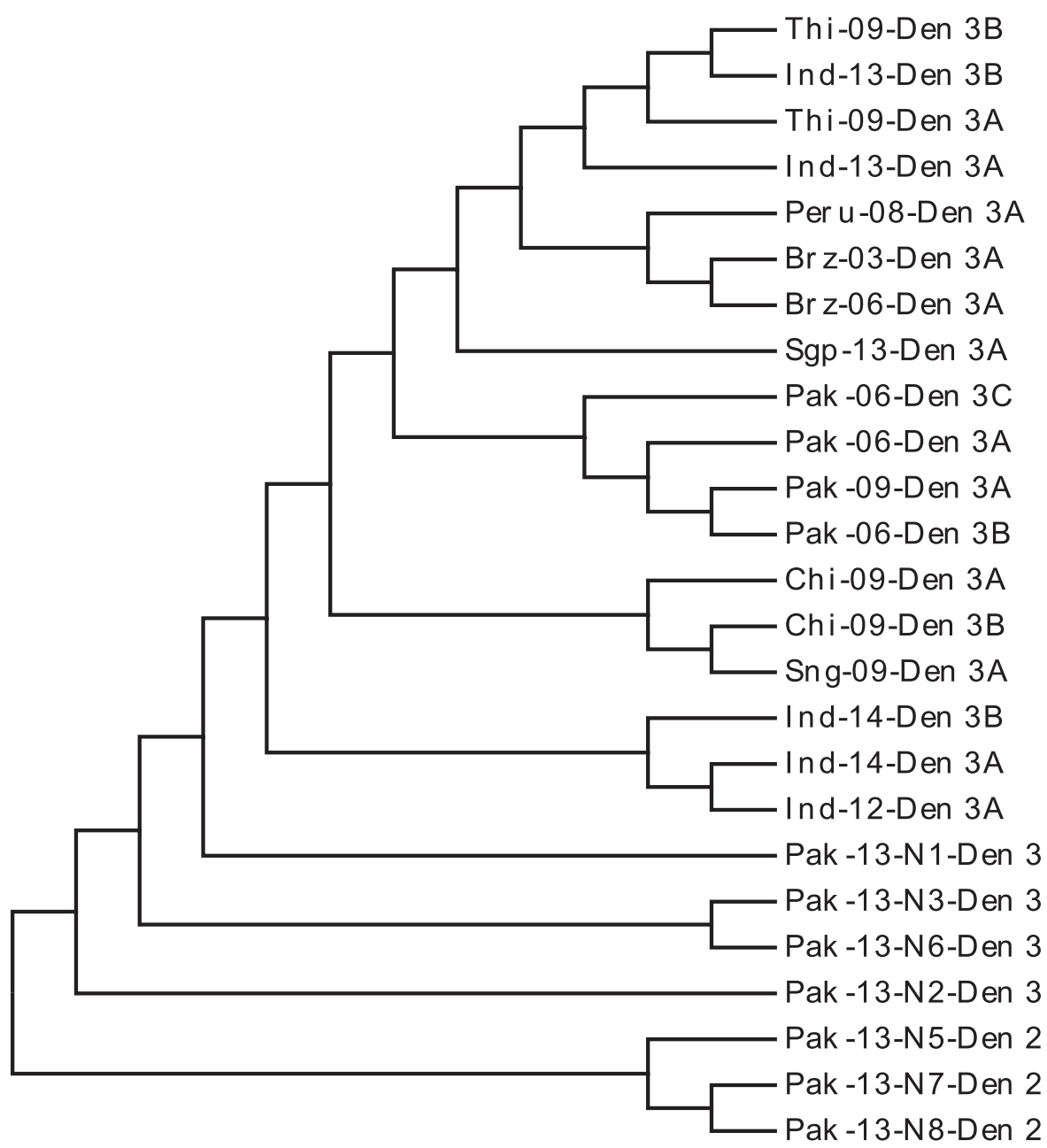

Fig. 3. Phylogenetic analysis of dengue virus serotype 3 based on C-prM regions corresponding to nt103-504 (401 bp) of the isolates in Lahore region in 2013. The four isolates of DENV-3 were aligned with reference sequences retrieved from GenBank. The tree was constructed by neighbor joining method using Mega 5.2 software. ${ }^{19}$

Thirty-nine NS1 positive samples were used for molecular detection and serotyping of dengue virus in Pakistan through RT-PCR and viral RNA was detected in only $8(20.5 \%)$ samples. The results of this study were in consistence with the previous studies that isolated viral RNA in $20(17.5 \%)$ out of 114 samples [16]. The reason of this low frequency of detection was that patients in this area tended to visit hospital after four or five days of fever, while high viremia levels in serum were found during first three to five days of fever. Previous studies reported dengue virus serotype 2 was the most prevalent in Pakistan, while the dominant serotype in our study was serotype 3, followed by serotype 2 and 1. genetic analysis of these samples revealed that Pakistani dengue virus serotype 1 was unique and formed a separate clad from dengue virus serotype- 1 isolate reported from Pakistan in 2006. It closely resembled Sri-Lankan strain that caused outbreak in 2012 [21] and it has similarity around 99\% with dengue virus strains circulating in Singapore in 2012 and 2013 and China in 2014 [22, 23]. This dengue virus strain most probably introduced into Pakistan from Sri Lanka. Pakistani dengue virus serotype 2 isolates formed a separate cluster near the strains which was reported in dengue virus outbreaks of 2008 and 2009 along with Indian and Singaporean strain reported in 2013 [16, 24]. Dengue virus 3 isolates also clustered together near a clad of Indian isolates which were reported in 2012 and 2014 outbreaks of dengue in India. The genetic analysis clearly indicated that in 2013 outbreak of dengue in Pakistan, three serotypes of dengue virus i.e. 1,2 and 3 were involved which might be introduce into Pakistan from Sri Lanka, Singapore and India respectively and can be avoided by proper quarantine measures and control of trade between countries.

\section{Conclusions}

Early diagnosis of disease can be helpful for the proper ant timely management of the patients. Diagnostic frequency can be increased by detecting NS1 antigen along with dengue specific IgM and IgG 
antibodies. According to this study both serological techniques (ICT and ELISA) were comparable. Therefore, immunochromatographic test devices can be used for screening of dengue patients in epidemic region as it is rapid and cost effective compared to ELISA method. Molecular detection and serotyping of dengue virus by RT-PCR was performed on acute phase sera. This study highlighted that 3 serotypes namely DENV-1, DENV-2 and DENV-3 were prevalent in our region. The most dominant serotype was DENV-3.

\section{Conflicts of Interest}

The authors declare no conflict of interest.

\section{References}

1. MULLER D.A., DEPELSENAIRE A.C., YOUNG P.R. Clinical and Laboratory Diagnosis of Dengue Virus Infection. J. Infec. Dis. 215 (2), 89, 2017.

2. TASLIM M., RSUNAN A.A., ISHAK H., NASIR S., USMAN A.N. Diversity of Dengue Virus Serotype in Endemic Region of South Sulawesi Province. Hindawi J. Trop. Med. Article ID 9682784, 1, 2018.

3. SHEPARD D.S., COUDEVILLE L., HALASA Y.A., ZAMBRANO B., DAYAN G.H. Economic impact of dengue illness in the Americas. Am. J. Trop. Med. Hyg. 84 (2), 200, 2011.

4. KONONGOI L., OFULA V., NYUNJA A., OWAKA S., KOKA H., MAKIO A., KOSKEI E., EYASE F., LANGAT D., RANDAL J., SCHOEPP R.J., ROSSI C.A., NJERU J., Coldren R., SANG, R. Detection of dengue virus serotypes 1, 2 and 3 in selected regions of Kenya: 2011-2014. Virol. J. 13, 182, 2016.

5. MURRAY N.E.A., QUAM M.B, WILDER-SMITH A. Epidemiology of dengue: Past, present and future prospects. Clin. Epidemiol. 5, 299, 2013.

6. FEREDE G., TIRUNEH M., ABATE E., WONDIMENEH Y., GADISA E., HOWE R., ASEFFA A., TESSEMA B. A study of clinical, hematological, and biochemical profiles of patients with dengue viral infections in Northwest Ethiopia: implications for patient management. BMC Infect. Dis. 18, 1, 2018.

7. SHARMA S., TANDEL K., DANWE S., BHATT P., DASH P.K., RANJANK P., RATHI R., GUPTA R.M., PARI M.M. Simultaneous detection and serotyping of dengue infection using single tube multiplex CDC Dengue Real-Time RT-PCR from Indi. Virus Dis. 29, 40, 2018.

8. NISHTAR S., BOERMA T., AMJAD S., ALAM A.Y, KHALID F. Pakistan's health system: performance and prospects after the $18^{\text {th }}$ Constitutional Amendment. Lancet. 381, 2193, 2013.

9. HUMAYOUN M.A., WASEEM T., JAWA A.A., HASHMI M.S., AKRAM J. Multiple dengue serotypes and high frequency of dengue hemorrhagic fever at two tertiary care hospitals in Lahore during the 2008 dengue virus outbreak in Punjab, Pakistan. Int J Infect Dis. Suppl 3:e54-9. doi: 10.1016/j.ijid.2009.10.008. Epub 2010 Feb 19. PMID: 20171916, 2010.
10. RASHEED S.B., BUTLIN RK, BOOTS M. A review of dengue as an emerging disease in Pakistan. Pub. Health. 127, 11, 2013.

11. World Health Organization (WHO). Dengue hemorrhagic fever: diagnosis, treatment, prevention and control. Geneva, 1997.

12. ARYA S., AGARWAL N. NS1 detection in addition to new dengue antibody assay with differential detection of $\operatorname{IgG}$ and Ig antibodies. Clin Biochem. 43, 537, 2010.

13. ANDRIES A.C., DUONG V., NGAN C., ONG S., HUY R. Field Evaluation and Impact on Clinical Management of a Rapid Diagnostic Kit That Detects Dengue NS1, Ig and IgG. Plos Negl Trop Dis. 6 (12), 2012.

14. INNIS B.L., NISALAK A., NIMMANNITYA S., KUSALERDCHARIYA S., CHONGSWASD I.V., SUNTAYAKORN S. An enzyme-linked immunosorbentassay to characterize dengue infections where dengue and Japanese encephalitis co-circulate. Am. J. Trop. Med. Hyg. 40, 418, 1989.

15. SHU P.Y., HUANG J.H. Current advances in dengue diagnosis. Clin. Diagnos. Lab. Immunol. 11(4), 642, 2004.

16. FATIMA Z., IDREES M., BAJWA M.A., TAHIR Z., ULLAH O., ZIA M.Q., HUSSAIN A., AKRAM M., KHUBAIB B., AFZAL S. Serotype and genotype analysis of dengue virus by sequencing followed by phylogenetic analysis using samples from three mini outbreaks-2007-2009 in Pakistan. BMC Microbiol. 11, 200, 2011.

17. VOGELSTEIN B., GILLESPIE D. Preparative and analytical purification of DNA from agarose. Proc. Natl. Acad. Sci. USA. 76, 615, 1979.

18. HALL T.A. BioEdit: a user-friendly biological sequence alignment editor and analysis program for Windows. Nucl. Acids Symp. Ser. 41, 95, 1999.

19. SAITOU N., NEI M. The neighbor-joining method: A new method for reconstructing phylogenetic trees. Mol. Bio. Evol. 4, 406, 1987.

20. SCOTT T.W, AND TAKKEN W. Feeding strategies of anthropophilic mosquitoes result in increased risk of pathogen transmission.Trends Parasitol. 28 (3), 114, 2012.

21. BODINAYAKE C.K., TILLEKERATNE L.G., NAGAHAWATTE A, DEVASIRI V., ARACHICHI W.K., STROUSE J.J., SESSIONS O.M., KURUKULASOORIYA R., UEHARA A., HOWE S. ON, X.M. Emergence of Epidemic Dengue-1 Virus in the Southern Province of Sri Lanka. Plos. Negl. Trop. Dis. 10 (10), 2016.

22. SUNG C., WEI, Y., WATANABE S., LEE H.S., KHOO Y.M., FAN L., RATHORE A.P., CHAN K.W.K., CHOY M.M., KAMARAJ U.S., SESSIONS O.M. Extended evaluation of virological, immunological and pharmacokinetic endpoints of CELADEN: a randomized, placebo-controlled trial of celgosivir in dengue fever patients. Plos. Negl. Trop. Dis. 10 (8), 2016.

23. WANG W., YU B., LIN X.D., KONG D.G., WANG J., TIAN J.H., LI M.H., HOLMES E.C., ZHANG Y.Z. Reemergence and autochthonous transmission of dengue virus, Eastern China, 2014. Emerging infectious diseases, 21 (9), 1670, 2015.

24. DAS, B., DAS, M., DWIBEDI, B., KAR, S.K., HAZRA, R.K. Molecular investigations of dengue virus during outbreaks in Orissa state, Eastern India from 2010 to 2011. Infect. Genet. Evol. 16, 401, 2013. 